\author{
ANNALS OF “DUNAREA DE JOS” UNIVERSITY OF GALATI \\ MATHEMATICS, PHYSICS, THEORETICAL MECHANICS \\ FASCICLE II, YEAR X (XLI) 2018, No. 2
}

Article DOI: $\underline{\text { https://doi.org/10.35219/ann-ugal-math-phys-mec.2018.2.10 }}$

\title{
OBSERVATIONS OF JETS IN THE MAGNETOSHEATH: FIRST RESULTS BASED ON CLUSTER DATA ANALYSIS
}

\author{
Simona Condurache-Bota ${ }^{1}$, Mirela Voiculescu ${ }^{1 *}$, Emilian Danila ${ }^{1}$, Marius \\ Echim $^{2,3}$ \\ ${ }^{I}$ Faculty of Sciences and Environment, Dunarea de Jos University of Galati, \\ 111 Domneasca Street, 800201, Galati, Romania, \\ scondurache@ugal.ro; ․mirela.voiculescu@ugal.ro; Emilian.Danila@ugal.ro \\ ${ }^{2}$ Institute of Space Science, Atomistilor 409, Magurele, Ilfov \\ ${ }^{3}$ Royal Belgian Institute of Space Aeronomy, Avenue Circulaire 3, 1180 Bruxelles, Begium
}

\begin{abstract}
The magnetosphere can be seen as a shield which protects the Earth from the impact of of the solar wind, which is a flux of charged particles originating from the Sun. Nevertheless, some of these particles penetrate the terrestrial atmosphere, and contribute to solar terrestrial events like e.g. auroras or, in extreme cases, disruption of satellite communications. It was recently discovered that the magnetosheath, i.e. the region formed at the interface between the solar wind and the magnetosphere, is the place where turbulent plasma jets are formed. This paper presents initial results of a data survey of such ion plasma jets detected in the magnetosheath in 2008. The study uses data provided by CLUSTER the magnetospheric mission of the European Space Agency (ESA)
\end{abstract}

Keywords: solar wind, magnetosheath, ion plasma jets, Cluster spacecraft mission

\section{INTRODUCTION}

The Sun is the origin of a flux of particles, mainly made of electrons and protons, known as the solar wind. It originates from the Sun's upper corona and it exhibits variable, supersonic speed (between 400-1200 km/s), densities of the order of $10 \mathrm{~cm}^{-3}$ and temperatures of the order of $100 \mathrm{eV}$ and. The flux of particles is embedded in a magnetic field, known as the Interplanetary Magnetic Field [1-5].

The region dominated by the action of the terrestrial magnetic field is called the magnetosphere [6]; its upper limit is the magnetopause (see Fig. 1), where the total pressures of the terrestrial and of the solar wind magnetic field and plasma are balanced. The shape of the magnetosphere is strongly influenced by the solar wind which compresses it on the sunward side (as low as 6 to 10 Earth radii, i.e. about $60,000 \mathrm{~km}$ ), while it extends on the opposite direction in what is called the magnetotail. Given the variability of the solar activity, the properties of the solar wind vary as well, sometimes reaching sudden intensifications when the speed of the solar wind particles may reach $1000 \mathrm{~km} / \mathrm{s}$ and even more. Such enormous speeds induce the further compression of the sunward part of the magnetosphere at only $35,000 \mathrm{~km}$, which amplifies the influence of the solar wind on the terrestrial atmosphere [1-5]. The shape of the planetary magnetic field lines and their deformation induced by the solar wind creates narrow regions, called cusps (or polar cusps), above the terrestrial poles, where solar energetic particles manage to enter directly into the magnetosphere. Dynamic 
auroras are the result of these interactions, which also have the potential to severely affect communications, power lines and telecommunications as well as the satellite technology. These negative impacts of solar wind particles have driven the scientific interest to understand the physics of the interaction between the solar wind and the terrestrial magnetosphere.

The bow shock forms at the impact of the supersonic solar wind with the magnetospheric "obstacle". Downstream the bow shock the solar wind is decelerated to subsonic speed and forms the magnetosheath. The supersonic flux of electrons and protons is abruptly slowed down and induce turbulence similar to a sound shock wave when decelerated.

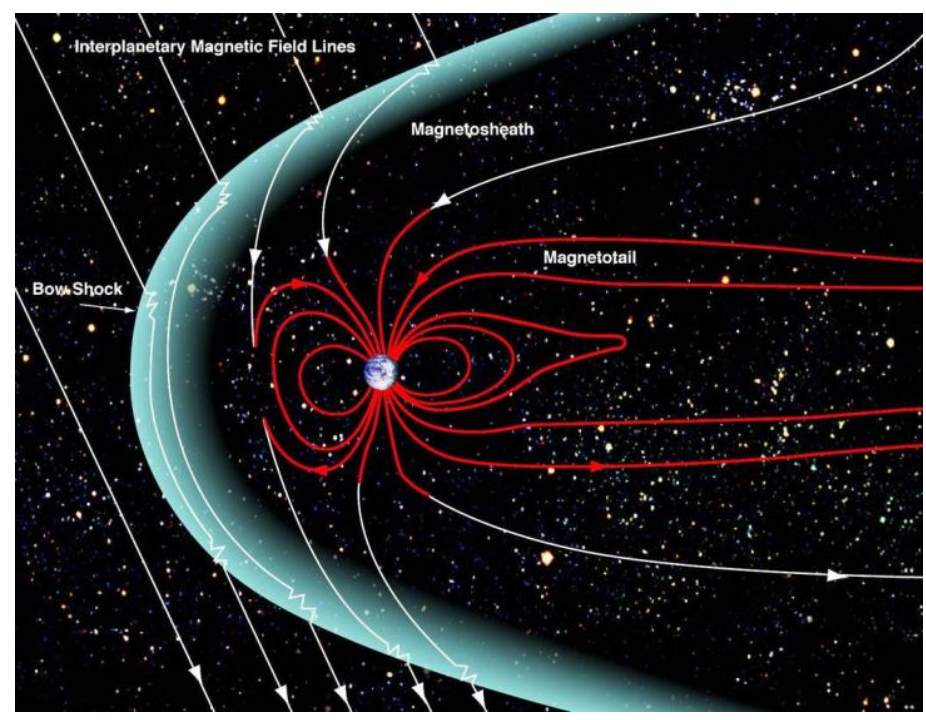

Figure 1. The solar wind interacting with

the terrestrial magnetosphere and its distinct regions [3].

The transition zone between the bow shock and the magnetopause is the magnetosheath, where plasma is highly turbulent compared to the upstream regions. The magnetosheath is the place where major changes happen while particles from the solar wind reach the magnetosphere.

The Cluster missions launched in 2000 and still working is an effort of the European Space Agency (ESA) and is designed to study the magnetospheric phenomena and the interaction with the solar wind $[1,2,5,7]$. The four Cluster satellites are able to collect three-dimensional information on how the solar wind interacts with the magnetosphere and affects near-Earth space and its atmosphere. It was recently found that transient enhancements of plasma dynamic pressure (due to increases of the speed and/or the density) are frequent in the terrestrial magnetosheath [9-15]. The four Cluster satellites along with other space missions, such as THEMIS (Time History of Events and Macroscale Interactions during Substorms) [8-10] and MMS (Magnetosphere Multiscale Mission) [10] provide solid experimental evidence for these events and contribute to the analysis of their main physical properties. These plasma entities were called "magnetosheath jets" [9-15] and are perhaps similar to what was called in the 80ties "magnetosheath plasmoids" or "blobs" or "plasma irregularities" [16-19]. It is believed these jets may have a strong impact on the magnetosphere depending on their orientation and dynamical pressure [e.g. 10]. Such protruding jets may contribute to the magnetosphereionosphere coupling and to trigger transient magnetospheric and ionospheric phenomena, like e.g. throat-type auroras [8-14].

The assessment of such transient intense plasma jets and their properties, as well as the effect on the magnetosphere and, further down, on the ionosphere, is still ongoing research. The identification of jets is still discussed whether jets should be defined based only on increased plasma density relatively to the "normal" magnetosheath or whether the plasma speed increase should be mainly considered, or if this increase relates to the full speed or only Sun-Earth component, etc. 
We present here the first results of a study aiming to investigate magnetosheath plasma jets within the terrestrial magnetosheath for January 2008, based on data provided by the Cluster spacecraft .

\section{DATA SOURCES AND METHODOLOGY OF ANALYSIS}

The Cluster mission was launched successfully in 2000 and recently it was confirmed by ESA that its lifetime is extended until 2022. It consists of 4 identical spacecraft flying in a tetrahedral configuration on elliptical polar orbits with $19000 \mathrm{~km}$ as perigee, $119000 \mathrm{~km}$ at apogee and a period of 57 hours. The four spacecraft are identical and carry 11 scientific instruments each, measuring the electron and ion plasma characteristics, including density, speed (with e.g. CIS-HIA, Cluster Ion Spectrometry; Hot Ion Analyzer [20]), electric and magnetic fields, with e.g. FGM - FluxGate Magnetometer [21] and EFW - Electric Field and Wave experiment [5,6,22]. Fig. 2 presents a configuration of all 4 Cluster spacecraft situated in the magnetosheath where ion plasma jets occur.

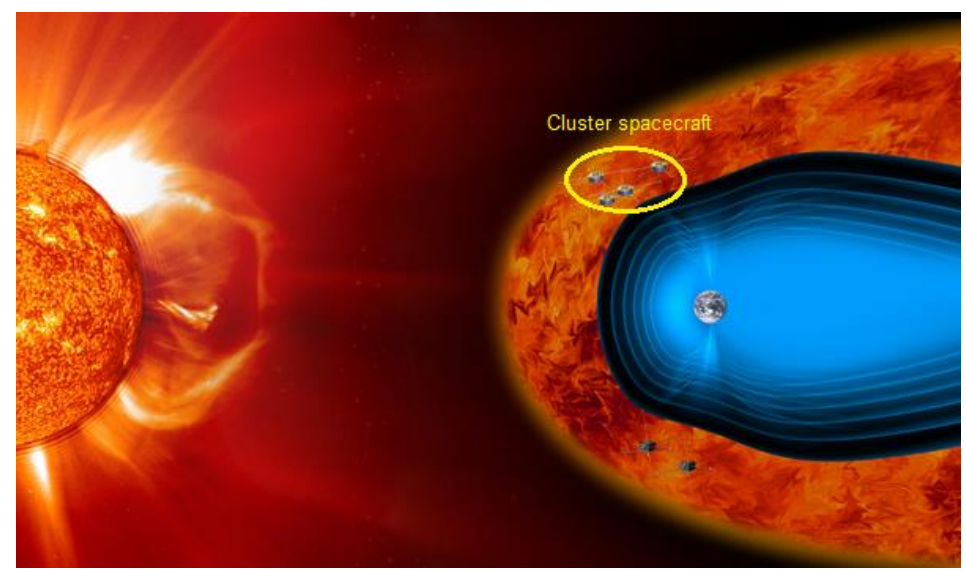

Figure 2. Situation of all 4 Cluster spacecraft in the terrestrial magnetosheath [15].

The data describing the plasma jets properties, namely the ion density and their velocity measured by Cluster are obtained from the AMDA database [23], which is "a versatile database for Space Physics", containing data from multiple space missions.
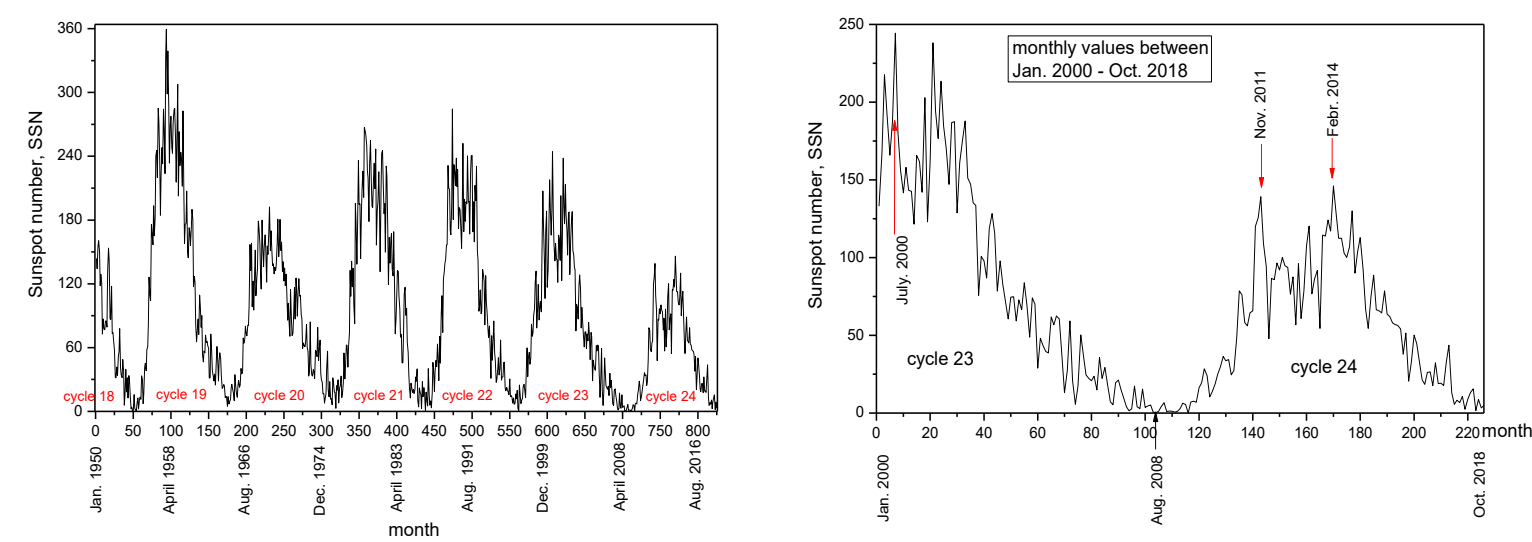

Fig. 3 Solar activity expressed by means of sunspot number (SSN) for the last 7 solar cycles (left) and in detail, for the last two solar cycles (right).

Several definitions and threshold values were proposed and studied so far for plasma jets within the magnetosheath [11]. In our study we use a data selection criterion based on the increase of the dynamic pressure with respect to the background level. The latter is estimated with a boxcar-type running average of dynamic pressure data over intervals of $15 \mathrm{~min}$. The dynamic pressure, $\mathrm{p}_{\mathrm{d}}$, was computed using the ion plasma speed, $v$, and not only its $\mathrm{v}_{\mathrm{x}}$ component, (the Sun-Earth direction), as: 


$$
\mathrm{p}_{\mathrm{d}}=\mathrm{m}_{\mathrm{p}} \cdot \mathrm{n} \cdot \mathrm{v}^{2}
$$

with $\mathrm{n}$ being the ion plasma density, expressed in $\mathrm{cm}^{-3}$, while $\mathrm{m}_{\mathrm{p}}=1.67 \cdot 10^{-27} \mathrm{~kg}$ is the proton mass.

\section{RESULTS AND DISCUSSION}

We choose 2008 for this first analysis of plasma jets within the magnetosheath is since it corresponds to the solar minimum at the end of solar cycle 23 (see Fig. 3) [22].

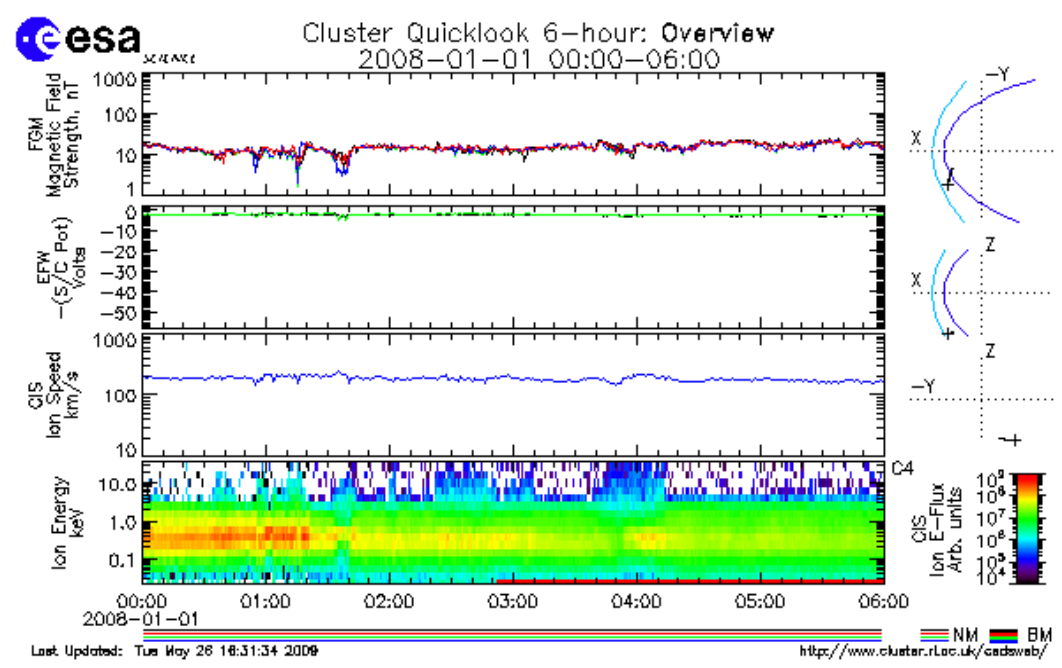

Figure 4. The position of the spacecraft (top, right), the magnetic field strength (upper plot), the intensity of the electric field ( $2^{\text {nd }}$ plot from the top), the ion speed ( $3^{\text {rd }}$ plot from the top) and the ion energy spectrum (the bottom plot) for Cluster 3 in the magnetosheath [25].

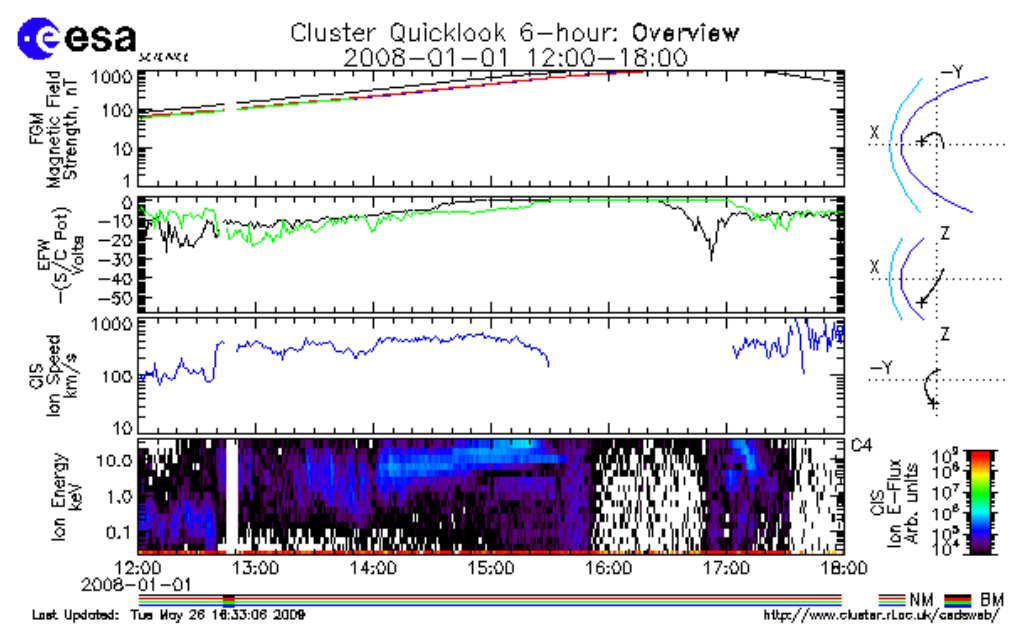

Figure 5. The position of the spacecraft (up, right), the magnetic field strength (upper plot), the intensity of the electric field ( $2^{\text {nd }}$ plot from the top), the ion speed ( $3^{\text {rd }}$ plot from the top) and the ion energy spectrum (the bottom plot) for Cluster 3 in the magnetosphere [25].

In this study we use data from Cluster 3 spacecraft provided by the AMDA database [23]. Prior to the analysis of jets we determined those time intervals when Cluster 3 was in the magnetosheath. These intervals are provided by the FP7 STORM project (Solar system turbulence, intermittency and multifractals) [24], while the UK database for the Cluster Mission [25] was used to assess the position of Cluster 3 with respect to the magnetosheath. 

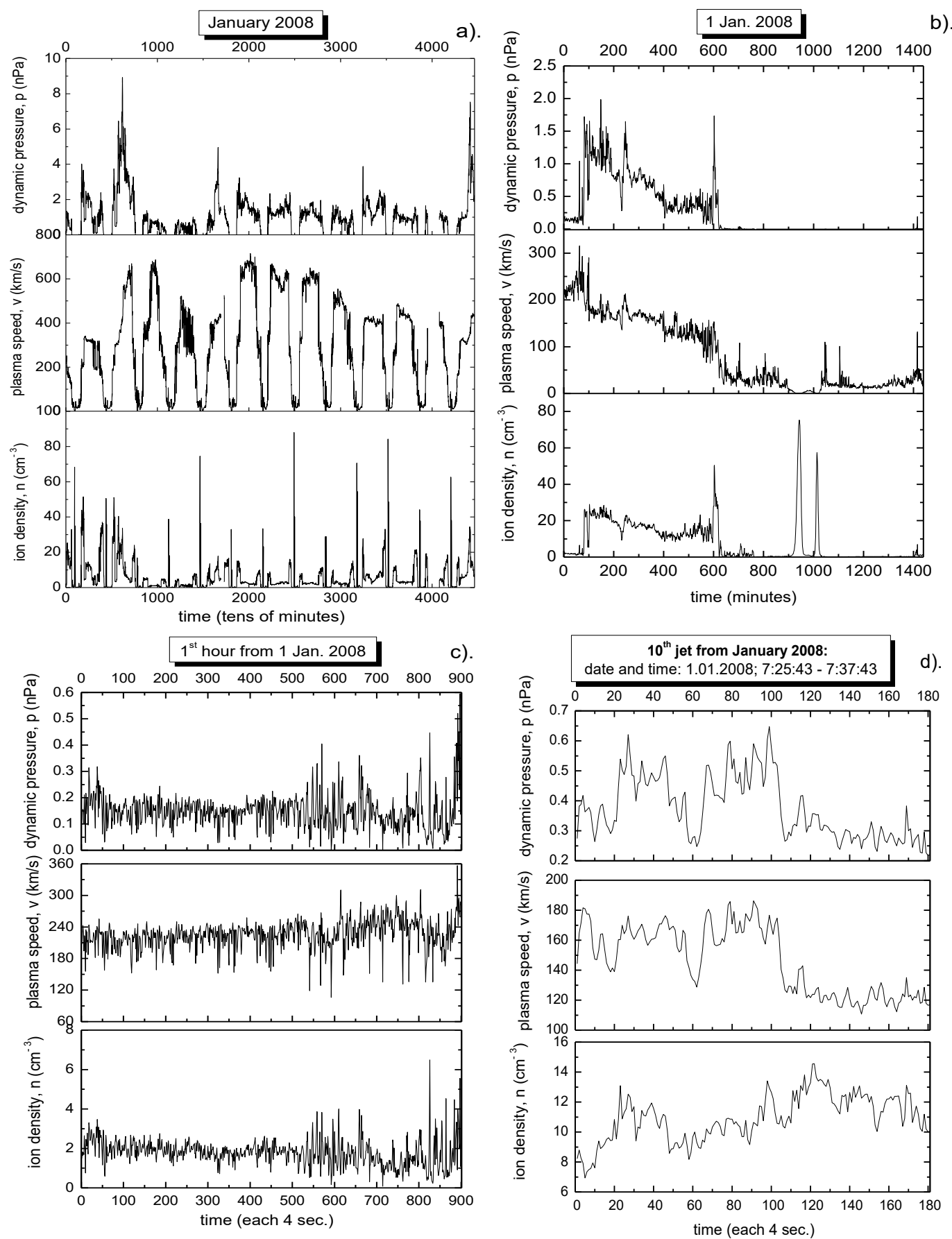

Fig. 6 The evolution of the ion density and the plasma speed as given by Cluster 3 and of the dynamic pressure, as computed from the previous parameters along different timescales: a. an entire month (January 2008); b. a day (1.01.2008); c. an hour; d. 12 minutes.

A number of 47 time intervals were found in January 2008, when the Cluster 3 spacecraft flew within the magnetosheath. Figures 4 and 5 present the position of the spacecraft (depicted as a small cross between the two curves delimitating the magnetosheath), the magnetic field strength, the intensity of the electric field, the ion speed and the ion energy spectrum for two positions of the Cluster 3 spacecraft: first, within the magnetosheath (Fig. 4) and then in the magnetosphere (Fig. 5) [23].

When comparing plots in Figs. 4 and 5, several relevant differences can be noticed, such as: 
- During the same day, i.e. 1.01.2008, Cluster 3 spacecraft flew within the magnetosheath between 0:00 and 6:00 A.M., but reached the magnetosphere between 12:00 and 18:00.

- While in the magnetosheath, Cluster 3 records low values for the magnetic field strength, slightly above $10 \mathrm{nT}$, as measured with the FGM instrument (top plot from Fig. 4). Then, when passing into the magnetosphere, the magnetic field strength increases abruptly to $100 \mathrm{nT}$ and $1000 \mathrm{nT}$, as characteristic for the magnetic region surrounding the Earth.

- The $2^{\text {nd }}$ plots from the top in Figs. 4 and 5 depict the spacecraft electrical potential, expressed in Volts. In both Figs. 4 and 5, this potential is negative or even close to zero, as expected, since the Cluster spacecraft are usually positive with respect to the plasma, such that the probe-to-spacecraft potential data is typically negative [26].

- The ion speed given in the $3^{\text {rd }}$ plot from above in Figs. 4 and 5 exhibits similar supersonic values, above $100 \mathrm{~km} / \mathrm{s}$. Interestingly, the speed in the magnetosheath is constant for this particular case, which is not a rule.

- The ion energy spectrum given as color-coded distributions in the bottom plots from Figs. 4 and 5 shows a higher flux in the magnetosheath than inside the magnetosphere. This is usually considered as relevant for the identification of the time intervals when a Cluster spacecraft enters the magnetosheath region, such that, within these intervals, ion plasma jets can be searched and studied.
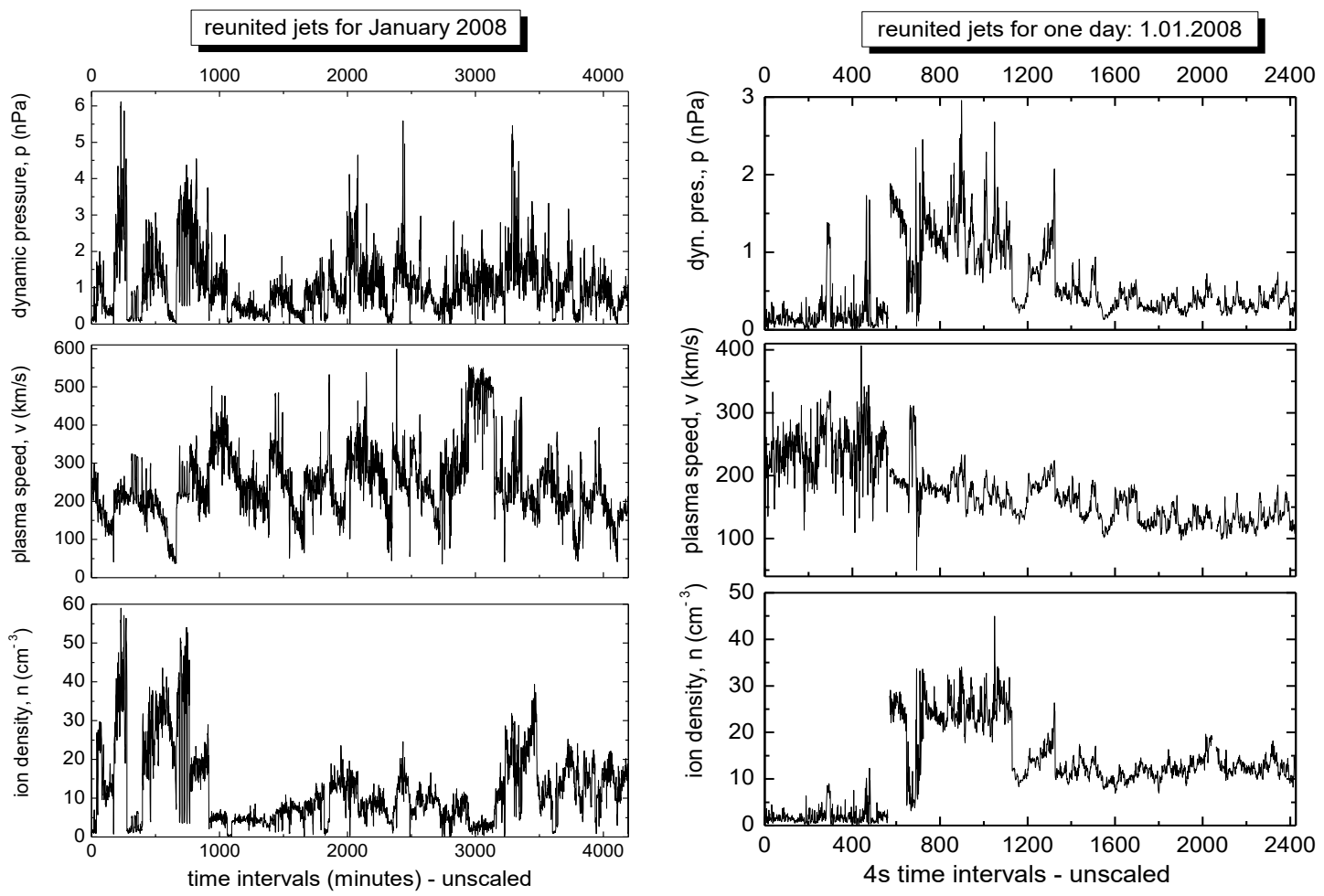

Figure 7. Reunited values for the ion density, plasma speed and dynamic pressure corresponding to sets of identified jet events for the entire month of

January 2008 (left-side plots) and, separately, for 1.01.2008 (right-side plots).

The temporal variation of plasma density, plasma speed and corresponding dynamic pressure for the entire month of January 2008 is presented in Fig. 6a, while Figs. 6b and 6c show the evolution of the same parameters on shorter timescales, namely during an entire day (1.01.2008 - in Fig. 6b) or merely for one hour, from 00:00 till 1:00 A.M. in 1.01.2008 (see Fig. 6c). The ion density regularly exhibits narrow peaks during an entire month (Fig. 6a), much higher than the background values and thus, easily detectable. During solely one day, as in 1.01.2008 (Fig. 6b), the plasma density ( $n$ ) strongly increases several times, while during only an hour, $n$ barely exhibits a narrow maximum. 

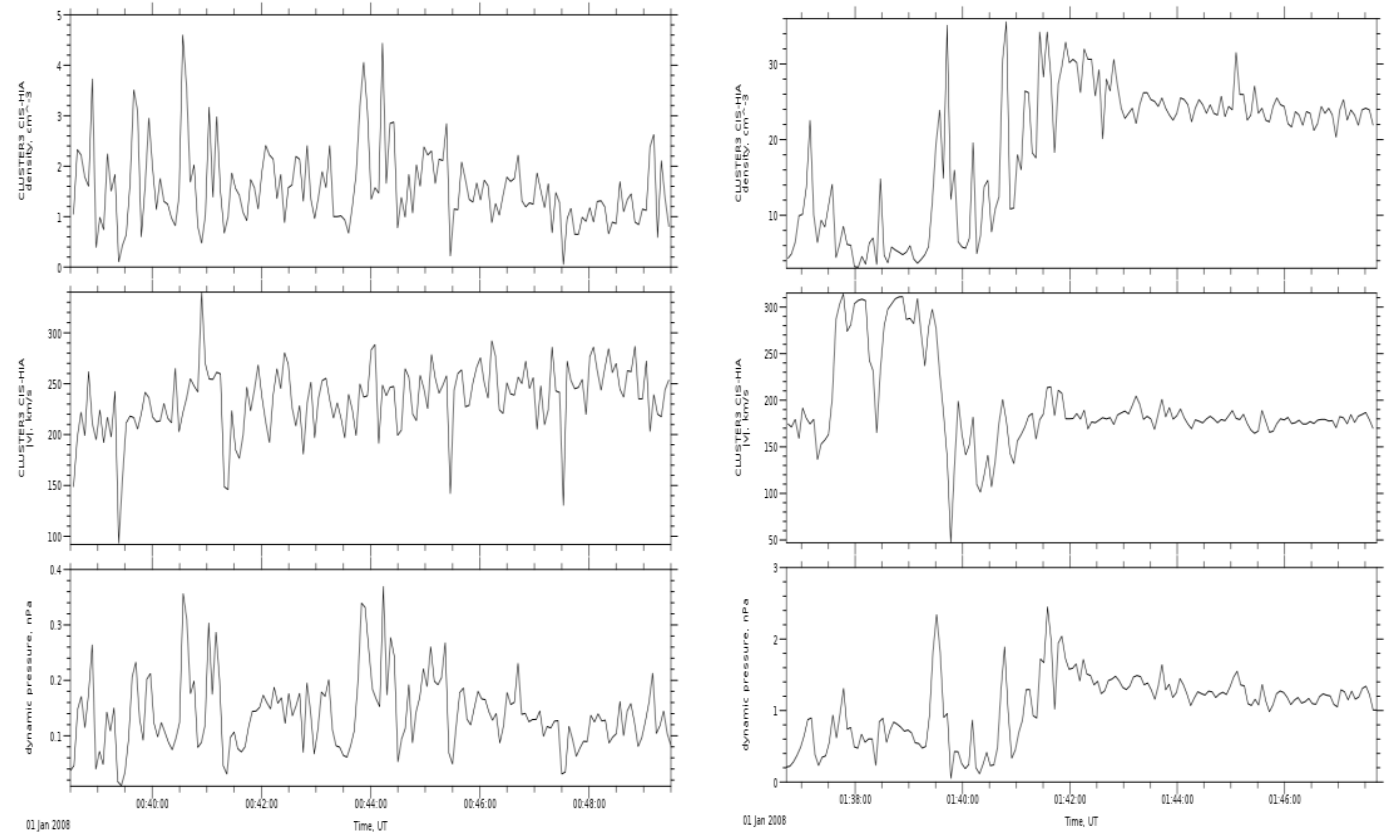

a. 1.01.2008, jet no. 1

b. 1.01.2008, jet no. 3
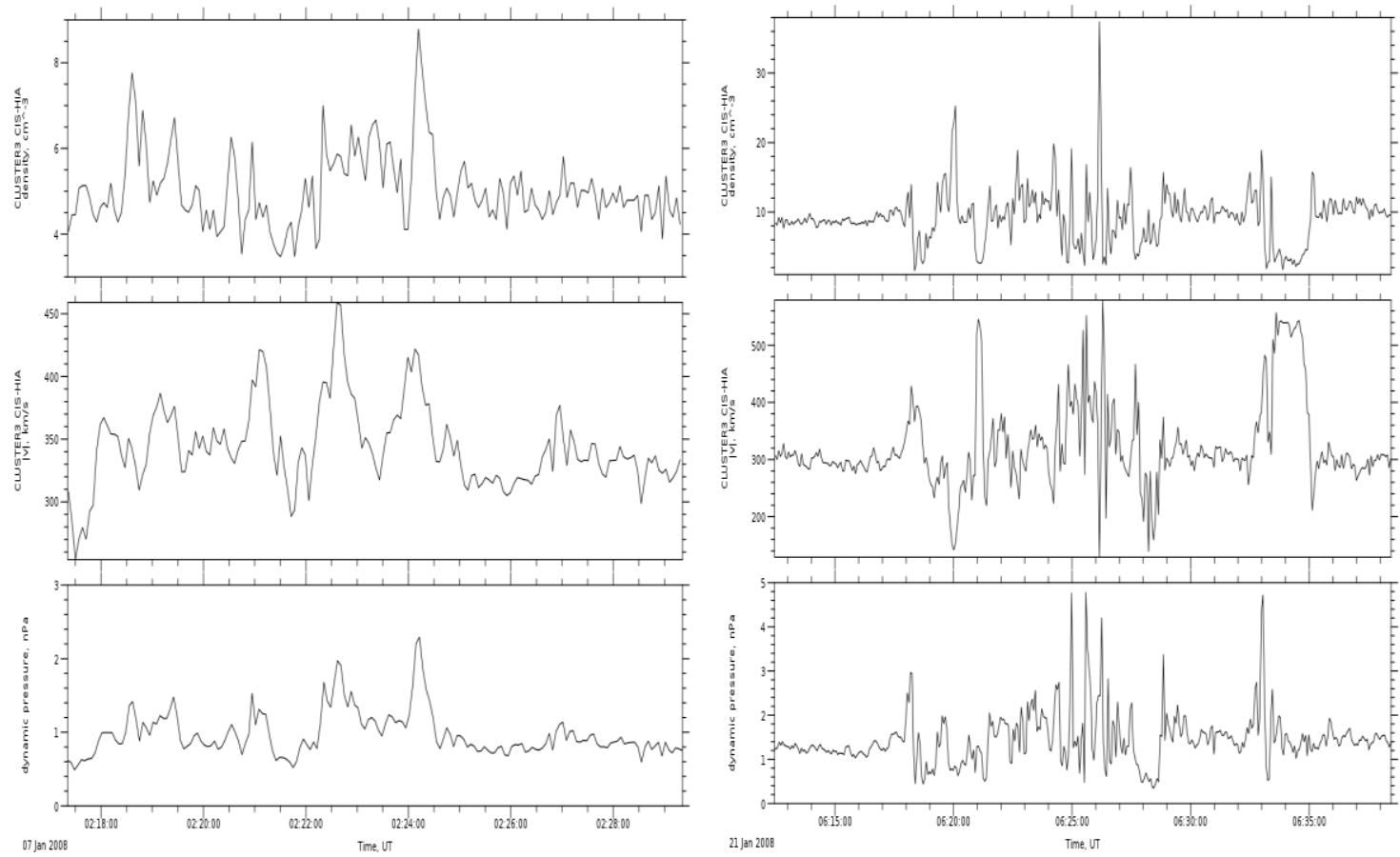

c. 1.01.2008, jet no. 50

d. 1.01.2008, jet no. 150

Figure 8. Temporal variation of the ion density (top plots), plasma speed (middle plots) and dynamic pressure (bottom plots) corresponding to 4 individual jets from 1.01.2008

The plasma speed, v, have an oscillatory variation during the selected month (Fig. 6a), with large variations from $100 \mathrm{~km} / \mathrm{s}$ up till $700 \mathrm{~km} / \mathrm{s}$, which is explained by the periodic movement of the spacecraft in and out of the solar wind (where the speed is highest). On a daily and an hourly bases, these oscillations are less clear (Figs. 6b and 6c) since the spacecraft position does not change much. The changes in speed/density induce an increase of the dynamic pressure. This is seen in Fig. $6 \mathrm{~d}$ for the 12 minutes interval during 1.01.2008, when two plasma jets were identified. 
The dynamic pressure at different timescales, as given in the top plots from Fig. 6, changes in a specific manner, since it is influenced by the evolution of both ion density and plasma speed. Such an abrupt increase of the dynamic pressure, $p_{d}$, can be noticeable only at shorter timescales, below one hour. This is because a jet event lasts from tens of seconds to few minutes at most [8], such that the variation of high dynamic pressure can be seen only during short periods.

Using AMDA, which also offers computing and plotting facilities, and the derived dynamic pressure, the jets were identified using a threshold value assumed for Pd. After a thorough series of attempts and trials, the threshold was fixed to:

$$
\mathrm{p}_{\mathrm{d} \text {,min }}=1.3 \cdot \mathrm{p}_{\mathrm{d} \text {, mean }}
$$

where $p_{d}$, mean is a running average over 600 seconds, with a 1 second time step [8, 11, 23, 27,28]. Consequently, a number of 456 plasma jets were identified for January 2008, for those time frames when the Cluster 3 spacecraft was situated within the magnetosheath (specified by the STORM database [24]).

The plots in Fig. 7 present the evolutions of the plasma density, its speed and the dynamic pressure for the sets of plasma jets identified for the entire month of January 2008 (left) and separately, only for 1.01.2008, respectively (right). The jet events were found to last from 30 seconds up till 7 minutes each, with an average of 89 seconds for the entire selected month. The plots in Fig. 7 show that the peak values of the dynamic pressure cover a large range and are due to ion density or plasma speed maxima, or both.

For the case study event chosen for data recorded on 1.01.2018, the dynamic pressure followed the pattern of the ion density, but this is not the case with every jet, as one can see in Fig. 8, where four jet events, are shown Jets nos. 1 and 3 present similar patterns for the dynamic pressure and the ion density, $n$, while jets nos. 50 and 150 are characterized by sudden increases of the ion density and the plasma speed, which are not necessarily correlated. During e.g. jet no.150, the plasma speed reaches a local maximum around 6:33 A.M., while the ion density decreases.

\section{CONCLUSIONS}

Plasma jet events or sudden increases of the dynamic pressure in the terrestrial magnetosheath were identified and analyzed using plasma and field data recorded by Cluster in January 2008. These events are most likely due to non-homogeneities induced downstream the bow-shock and not to variations of the solar wind-source $[8,11]$.

A deeper study of such jet events is of high interest, due to their potential of changing, even on a short scale, magnetosphere and ionosphere properties. This paper presents the preliminary results of jet identification within the magnetosphere along only one month - January 2008, while the Sun was quiet. The study will be extended to larger time intervals. The first results successfully tested the methodology that can be now applied on routinely basis..

Acknowledgements: The VESS project (Far-reaching valorization of research experience in space and security, Project No. PN-III-P1-1.2-PCCDI-2017-0371, 18PCCDI/2018, funded by the Romanian Ministery of Research and Innovation, CCCDI-UEFISCDI, within PNCDI III) is acknowledged for the financial support of the authors, while the AMDA database, the ESA Cluster mission, and the UK Cluster Data Centre and the SILS database are deeply thanked for their efforts and progress in science and technology and extended data provided. The STORM project is also acknowledged for providing the intervals when the Cluster 3 spacecraft laid in the magnetosheath.

\section{References}


[1] T. Horbury, Waves and turbulence in the solar wind, Imperial College London, 23 sept. 2003, ppt presentation.

[2] Wayne R. Keith, The Magnetosphere: Earth's Invisible Shield, Angelo State University, McMurry University, 1.02.2006.

[3] https://www.nasa.gov/mission pages/sunearth/multimedia/magnetosphere.html

[4] H. Hietala, T. V. Laitinen, K. Andreeova, R. Vainio, A. Vaivads, M. Palmroth, T. I. Pulkkinen, H.

E. J. Koskinen, E. A. Lucek, and H. Reme, Supermagnetosonic jets behind a collisionless quasiparallel shock, Physical Review Letters, 103, 245001, 2009.

[5] http://sci.esa.int/cluster/

[6] T. Gold, Motions in the magnetosphere of the Earth, Journal Geophys. Res., 64 (9), 1219-1224, 1959.

[7] J. M. Bosqued, T. D. Phan, I. Dandouras, C. P. Escoubet, H. Reme, A. Balogh, M.W. Dunlop, D. Alcayde, E. Amata, M.-B. Bavassano-Cattaneo, R. Bruno, C. Carlson, A. M. DiLellis, L. Eliasson, V. Formisano, L. M. Kistler, B. Klecker, A. Korth, H. Kucharek, R. Lundin, M. McCarthy, J. P. McFadden, E. Mobius, G. K. Parks, and J.-A. Sauvaud, Cluster observations of the high-latitude magnetopause and cusp: initial results from the CIS ion instruments, Annales Geophysicae, 19, 15451566, 2001.

[8] F. Plaschke, H. Hietala, and V. Angelopoulos, Anti-sunward high-speed jets in the subsolar magnetosheath, Ann. Geophys., 31, 1877-1889, 2013.

[9] A. V. Dmitriev, and A. V. Suvorova, Traveling magnetopause distortion related to a large-scale magnetosheath plasma jet: THEMIS and ground-based observations, J. Geophys. Res., 117, A08217, 2012.

[10] F. Plaschke, and H. Hietala, Plasma flow patterns in and around magnetosheath jets, Ann. Geophys., 36, 695-703, 2018.

[11] T. Karlsson, F. Plaschke, H. Hietala, M. Archer, X. Blanco-Cano, P. Kajdic, P.-A. Lindqvist, G. Marklund, and D. J. Gershman, Investigating the anatomy of magnetosheath jets - MMS observations, Ann. Geophys., 36, 655-677, 2018.

[12] D.-S. Han, Y. Nishimura, L. R. Lyons, H.-Q. Hu, and H.-G. Yang, Throat aurora: The ionospheric signature of magnetosheath particles penetrating into the magnetosphere, Geophys. Res. Lett., 43, 1819-1827, 2016.

[13] D.-S. Han, H. Hietala, X.-C. Chen, Y. Nishimura, L. R. Lyons, J.-J. Liu, H.-Q. Hu, and H.-G. Yang, Observational properties of dayside throat aurora and implications on the possible generation mechanisms, J. Geophys. Res. Space Physics, 122, 1853-1870, 2017.

[14] T. Karlsson, N. Brenning, H. Nilsson, J..-G. Trotignon, X. Vallières, G. Facsko, Localized density enhancements in the magnetosheath: Three-dimensional morphology and possible importance for impulsive penetration. Journal of Geophysical Research: Space Physics, American Geophysical Union/Wiley, 117, A03227 (17 p.), 2012.

[15] http://sci.esa.int/cluster/59947-cluster-measures-turbulence-in-earth-s-magnetic-environment/

[16] J. Lemaire, Plasmoid motion across a tangential discontinuity - With application to the magnetopause, J. Plasma Phys., 33, 425-436, 1985.

[17] G. Voitcu, M. Echim, Transport and entry of plasma clouds/jets across transverse magnetic discontinuities: Three-dimensional electromagnetic particle-in-cell simulations, J. Geophys. Res.: Space Phys., 121 (5), 4343-4361, 2016.

[18] Voitcu, Gabriel; Echim, Marius, Tangential deflection and formation of counterstreaming flows at the impact of a plasma jet on a tangential discontinuity, Geophys. Res. Lett., 44 (12), 5920-5927, 2017.

[19] Voitcu, Gabriel; Echim, Marius, Crescent-shaped electron velocity distribution functions formed at the edges of plasma jets interacting with a tangential discontinuity, Ann. Geophys., 36 (6), 1521$1535,2018$.

[20] H. Reme, J. M. Bosqued, J. A. Sauvaud, A. Cros, J. Dandouras, C. Aoustin, J. Bouyssou et al., The Cluster Ion Spectrometry (cis) Experiment, Space Sci. Rev., 79 (1/2), 303-350, 1997.

[21] A. Balogh, M. W. Dunlop, S. W. H. Cowley, D. J. Southwood, J. G. Thomlinson, K. H. Glassmeier, G. Musmann, H. Luhr, S. Buchert, M. H. Acuna, D. H. Fairfield, J. A. Slavin, W. 
Riedler, K. Schwingenschuh, M. G. Kivelson, The Cluster Magnetic Field Investigation, Space Sci. Rev., 79 (1/2), 65-91, 1997.

[22] G. Gustafsson, R. Bostrom, B. Holback, G. Holmgren, A. Lundgren, K. Stasiewicz, L. Ahlen, F. S. Mozer, D. Pankow, P. Harvey et al., The Electric Field and Wave Experiment for the Cluster Mission, Space Sci. Rev., 79 (1/2), 137-156, 1997.

[23] http://amda.irap.omp.eu

[24] http://www.storm-fp7.eu

[25] http://www.cluster.rl.ac.uk/

[26] P.-A. Lindqvist, C. Cully, Y. Khotyaintsev, and the EFW team, User Guide to the EFW measurements in the Cluster Active Archive (CAA), Doc. No. CAA-EST-UG-EFW, Issue: 3.1, 201104-28, 1-26, 2011.

[27] M. O. Archer, T.S. Horbury, J. P. Eastwood, J. M. Weygand, and T. K. Yeoman, Magnetospheric response to magnetosheath pressure pulses: A low-pass filter effect, J. Geophys. Res., 118, 54545466, 2013.

[28] T. Karlsson, A. Kullen, E. Liljeblad, N. Brenning, H. Nilsson, H. Gunell, and M. Hamrin, On the origin of magnetosheath plasmoids and their relation to magnetosheath jets, J. Geophys. Res., 120, 7390-7403, 2015. 